\title{
Cycles and Bipartite Graph on Conjugacy Class of Groups
}

\author{
BiJAN TAERI $(*)$
}

\begin{abstract}
Let $G$ be a finite non abelian group and $B(G)$ be the bipartite divisor graph of a finite group related to the conjugacy classes of $G$. We prove that $B(G)$ is a cycle if and only if $B(G)$ is a cycle of length 6 and $G \cong A \times S L_{2}(q)$, where $A$ is abelian, and $q \in\{4,8\}$. We also prove that if $G / Z(G)$ simple, where $Z(G)$ is the center of $G$, then $B(G)$ has no cycle of length 4 if and only if $G \cong A \times S L_{2}(q)$, where $q \in\{4,8\}$.
\end{abstract}

\section{Introduction and results.}

Assigning a graph to a group is one of the interesting tools in investigating the structure of groups. One of the extensively studied graphs is the graph related to conjugacy class sizes. In fact various graphs related to conjugacy class sizes have been defined and studied. For a survey on the subject see [15]. The bipartite divisor graph is recently introduced for a set of arbitrary integers in [11]. The bipartite graph divisor for the set of conjugacy classes is the object of [3].

Let us introduce some notation. Let $G$ be a finite group $x$ be any element of $G$. We denote by $x^{G}=\left\{x^{g} \mid g \in G\right\}$, where $x^{g}=g^{-1} x g$, the conjugacy class of $x$ in $G$ and by $\operatorname{cs}(G)=\left\{\left|x^{G}\right| \mid x \in G\right\}$ the set of the conjugacy class sizes of $G$. Let $\operatorname{cs}^{*}(G)=\operatorname{cs}(G) \backslash\{1\}$ be the set of sizes of the noncentral classes of $G$. Recall that the prime vertex graph $\Delta:=\Delta(G)$ is the graph with vertex set $V(\Delta)=\rho(G)=\bigcup_{n \in \operatorname{cs}(G)} \pi(n)$, where $\pi(n)$ is the set of prime divisors of $n$, and edge set $E(\Delta)=\{\{p, q\} \mid p q$ divides some $n \in \operatorname{cs}(G)\}$. The common divisor graph $\Gamma:=\Gamma(G)$ is the graph with vertex set $V(\Gamma)=\operatorname{cs}^{*}(G)$, and two vertices $n, m \in \operatorname{cs}^{*}(G)$ are joined if

(*) Indirizzo dell'A.: Department of Mathematical Sciences, Isfahan University of Technology, Isfahan 84156-83111, Iran.

E-mail: b.taeri@cc.iut.ac.ir 
$\operatorname{gcd}(m, n) \neq 1$. The bipartite divisor graph $B(G)$, studied in [3], is the graph with vertex set the disjoint union $\rho(G) \cup \operatorname{cs}^{*}(G)$ and with edge set $\left\{\{p, n\} \mid p \in \rho(G), n \in \operatorname{cs}^{*}(G)\right.$ and $p$ divides $\left.n\right\}$.

In [3] the authors, among other things, characterized finite groups $G$ such that $B(G)$ is a path of length 5 . In this paper we consider a similar problem and characterize finite groups $G$ such that $B(G)$ is cycle.

THeORem 1. Let $G$ be a finite group such that $B(G)$ is a cycle. Then $G=A \times S$, where $A$ is abelian, and $S \cong S L_{2}(q), q=4,8$. Consequently $B(G)$ is a cycle if and only if $B(G)$ is the 6-cycle $2-12-3-15-5-20-2$ and $G \cong A \times S L_{2}(4)$, or is the 6-cycle $2-72-3-63-7-56-2$ and $G \cong A \times S L_{2}(8)$, where $A$ is abelian.

In [6] the authors studied the groups $G$ such that $\Gamma(G)$ has no triangle. In this paper we study the groups $G$ such that $B(G)$ has no square. We prove that

THeOREm 2. Let $G$ be a finite group such that $G / Z(G)$, where $Z(G)$ denotes the center of $G$, is simple. Then $B(G)$ has no cycle of length 4 if and only if $G=A \times S$, where $A$ is abelian, and $S \cong S L_{2}(q), q=4,8$.

\section{Finite groups with cyclic bipartite divisor graphs.}

We use the following lemma which contains some well known facts.

Lemma 1. Let $G$ and $H$ be groups.

(i) $\operatorname{cs}(G \times H)=\{a b \mid a \in \operatorname{cs}(G), b \in \operatorname{cs}(H)\}$.

(ii) If $x, y \in G$ commute and have coprime orders, then $C_{G}(x y)=$ $=C_{G}(x) \cap C_{G}(y)$ and so $\left|x^{G}\right|$ and $\left|y^{G}\right|$ divide $\left|(x y)^{G}\right|$.

(iii) If $N$ is a normal subgroup of $G$ and $x \in N$, then $\left|x^{N}\right|$ divides $\left|x^{G}\right|$. Also if $y \in G$, then $\left|(y N)^{G / N}\right|$ divides $\left|y^{G}\right|$.

All of the cycles are 2-regular graphs and bipartite 2-regular graphs are cycles. If $B(G)$ is 2-regular then every noncentral conjugacy class has exactly 2 prime divisors. Thus we can use a result of Casolo.

Lemma 2 (Proposition 1 of [4]). Let $G$ be a non-solvable group with $\sigma^{*}(G)=2$, where $\sigma^{*}(G)=\max \left\{\left|\pi\left(g^{G}\right)\right|: g \in G\right\}$. Then $G=A \times S$, where $A$ is abelian and $S$ is isomorphic to either $P S L_{2}(4)$ or $P S L_{2}(8)$. 
A non abelian group $G$ is an $F$-group if for all non central elements $x, y$, $C_{G}(x) \leq C_{G}(y)$ implies that $C_{G}(x)=C_{G}(y)$. The concept of $F$-group is essential in this paper. The structure of an $F$-groups is given by Rebmann.

Lemma 3 (See [17]). Let $G$ be a non abelian group. Then $G$ is an F-group if and only if it is one of the following types:

(1) G has a normal abelian subgroup of prime index.

(2) $G / Z(G)$ is a Frobenius group with Frobenius kernel $L / Z(G)$ and Frobenius complement $K / Z(G)$ with $K$ and $L$ abelian.

(3) $G / Z(G)$ is a Frobenius group with Frobenius kernel $L / Z(G)$ and Frobenius complement $K / Z(G)$ with $K$ abelian, $Z(L)=Z(G)$, $L / Z(G)$ has prime power order and $L$ is an F-group.

(4) $G / Z(G) \cong S_{4}$ and if $V / Z(G)$ is the Klein four-group in $G / Z(G)$, then $V$ is not abelian.

(5) $G=P \times A$ where $P$ is an F-group of prime power order and $A$ is abelian.

(6) $G / Z(G) \cong P S L_{2}\left(p^{n}\right)$ or $P G L_{2}\left(p^{n}\right), G^{\prime} \cong S L_{2}\left(p^{n}\right)$, where $G^{\prime}$ is the derived subgroup of $G$, $p$ a prime, $p^{n}>3$.

(7) $G / Z(G) \cong P S L_{2}(9)\left(\cong A_{6}\right)$ or $P G L_{2}(9)$, and $G^{\prime}$ is isomorphic to the Schur cover of $P S L_{2}(9)$.

In what follows we find a characterization of finite groups with cyclic bipartite graphs.

Proof of Theoerem 4. If $G$ non-solvable then by Lemma 2, $G \cong A \times S L_{2}(q)$, where $i=4,8$, and $A$ is abelian. If $G \cong A \times S L_{2}(4) \cong$ $\cong A \times A_{5}$, then since $\operatorname{cs}^{*}\left(A_{5}\right)=\{12,15,20\}, \quad B(G)$ is the 6 -cycle $2-12-3-15-5-20-2$. If $G \cong A \times S L_{2}(8)$, then since $\operatorname{cs}^{*}\left(S L_{2}(8)\right)=$ $=\{56,63,72\}, B(G)$ is the 6 -cycle $2-72-3-63-7-56-2$.

Now we show that $G$ cannot be solvable. By the way of contradiction, suppose that $G$ is solvable and $B(G)$ is a cycle. Thus, for all non-central $g \in G$, we have $\pi\left(g^{G}\right)=2$. First note that by Corollary C of [9], if $\operatorname{cs}^{*}(G)=\{m, n\}$ and $\operatorname{ged}(m, n) \neq 1$, then either $m$ or $n$ is a prime power and thus $B(G)$ cannot be a cycle of length 4 . By [4, Theorem 3.7] we have $|V(\Delta(G))| \leq 4$. If $|V(\Delta(G))|=2$, then since $B(G)$ is 2-regular, $B(G)$ is a cycle of length 4, which is a contradiction. So $|V(\Delta(G))| \geq 3$. Also we know that $\pi(G / Z(G))=V(\Delta(G))$ (see for example [3, Corollary 7]), and so $3 \leq|\pi(G / Z(G))| \leq 4$. If $\left|\operatorname{cs}^{*}(G)\right|=2$, then $B(G)$ is a cycle of length 4 , which is impossible. Hence $\left|\operatorname{cs}^{*}(G)\right| \geq 3$. 
We claim that $G$ is an $F$-group. First note that if $c=p^{a} q^{b} \in \operatorname{cs}^{*}(G)$, with $a$ and $b$ positive, then for all $x \in G$ with $p \in \pi\left(x^{G}\right) \subseteq\{p, q\}$ we must have $\left|x^{G}\right|=p^{a} q^{b}$, since $B(G)$ is 2-regular. Let $x, y$ be two elements of $G$ such that $C_{G}(x) \leq C_{G}(y)$. We have to prove $C_{G}(x)=C_{G}(y)$. If $C_{G}(x)<C_{G}(y)$, then $\left|x^{G}\right|$ and $\left|y^{G}\right|$ are distinct and $\left|y^{G}\right|$ divides $\left|x^{G}\right|$, which is impossible. Hence $G$ is an $F$-group and so is one of the groups listed in Lemma 3. The groups listed in Lemma 3 (6) and (7) are non-solvable. Thus $G$ is one of the group listed in (1)-(5).

Suppose (1) holds. Then $G$ has an abelian normal subgroup $N$ of prime index $p$. We show that $\operatorname{cs}^{*}(G)=\{p, m\}$, for some $m$. Let $x$ be any non-central element of $G$. If $x \in N$, then since $N \leq C_{G}(x)<G$ and $|G / N|=p$, we have $\left|x^{G}\right|=p$. If $x \notin N$, then $G=N\langle x\rangle$ and so $m:=\left|x^{G}\right|=\left|N: C_{N}(x)\right|$. For any non-central element $y \in G$, there exists $a \in N$ such that $y=a x$. Therefore $C_{N}(x)=C_{N}(y)$ and hence $\left|y^{G}\right|=m$. Thus $\operatorname{cs}^{*}(G)=\{p, m\}$, a contradiction.

If (2) holds, then $\left|\operatorname{cs}^{*}(G)\right|=2$, a contradiction. (Recall that if $G$ is quasiFrobenius with abelian kernel and complement has exactly three conjugacy class sizes, see for example [16, Lemma 4.4]).

Suppose (3) holds. Let $x \in K \backslash Z(G)$. Since $C_{G}(x)=K$ we have $\left|x^{G}\right|=$ $=|G: K|=|L: L \cap Z(G)|$, which is a prime power, a contradiction.

If (4) holds, then $\pi(G / Z(G))=\pi\left(S_{4}\right)=\{2,3\}$, a contradiction.

If (5) holds, then $\operatorname{cs}^{*}(G)=\operatorname{cs}^{*}(P)$, a contradiction.

Thus $G$ cannot be solvable.

As mentioned above there is no group with $B(G)$ a cycle of length 4. Let $K_{m, n}$ be a complete bipartite graph with bipartite halves of sizes $m$ and $n$. Since $K_{2,2}$ is a cycle of length 4 the following question arises.

QUESTION 1. Is there a group $G$ with $B(G)=K_{m, n}$ ?

\section{Finite groups whose bipartite divisor graphs contain no cycle of length 4.}

In this section we investigate the structure of groups such that $B(G)$ contains no cycle of length 4 . We say that $G$ satisfies the one-prime power hypothesis, if $m, n \in \operatorname{cs}^{*}(G)$, then either $\operatorname{gcd}(m, n)=1 \mathrm{or} \operatorname{gcd}(m, n)$ is a power of a prime. The terminology is similar to the one-prime hypothesis introduced by Lewis [15] on character degrees. One can see easily that the graph $B(G)$ has no cycle of length 4 if and only if $G$ satisfies the one-prime power hypothesis: Suppose $B(G)$ has no cycle of length 4 and let $m, n \in \operatorname{cs}^{*}(G)$. If 
$p q$ divides $\operatorname{gcd}(m, n)$, then $p-m-q-n-p$ is a cycle of length 4 in $B(G)$, a contradiction. Thus $\operatorname{gcd}(m, n)=p^{a}$, for some prime $p$ and integer $a \geq 0$. Conversely if for all $m, n \in \operatorname{cs}^{*}(G), \operatorname{gcd}(m, n)$ is a prime power, then $G$ has no cycle of length 4 . In fact if $p-m-q-n-p$ is a cycle of length 4 in $B(G)$, then $p q$ divides $\operatorname{gcd}(m, n)$ and so $\operatorname{gcd}(m, n)$ is not a prime power.

Suppose that $G / Z(G) \cong S$, where $S$ is a group such that $S=S^{\prime}$. If $x$ is a non-central element of $G$, then $\left|x^{G}\right|=c a$, where $c=\left|(x Z(G))^{G / Z(G)}\right|$ and $a$ is a divisor of $|M(S)|(M(S)$ is the Schur multiplier of $S)$. To see this, let $D=\{g \in G \mid[g, x] \in Z(G)\}$, where $[g, x]=g^{-1} g^{x}$, be the pre-image of $C_{G / Z(G)}(x Z(G))$ in $G$. Since $C_{G}(x)$ is the kernel of the homomorphism $D \longrightarrow G^{\prime} \cap Z(G)$ with $d \mapsto[d, x]$, it follows that $\left|D / C_{G}(x)\right|$ divides $\left|G^{\prime} \cap Z(G)\right|$. By [18, Theorem 9.18 (1) and (6)], $\left|G^{\prime} \cap Z(G)\right|$ divides $|M(S)|$ and so $a:=|D / C|$ divides $|M(S)|$. Now $\left|x^{G}\right|=\left|G: C_{G}(x)\right|=$ $=|G / Z(G): D / Z(G)|\left|D / Z(G): C_{G}(x) / Z(G)\right|=c a$, as claimed.

By [10, Satz 25.7] we know that if $q \neq 9$ is odd, then $\left|M\left(P S L_{2}(q)\right)\right|=2$; if $q \neq 4$ is a power of 2 , then $\left|M\left(P S L_{2}(q)\right)\right|=1$; and $\left|M\left(P S L_{2}(4)\right)\right|=2$, $\left|M\left(P S L_{2}(9)\right)\right|=6$. We can use these information in the proof of the following lemma.

In the following lemma we also use the well known Burnside's $p^{\alpha}$ Lemma which states that a finite group which has a conjugacy class of a prime power size is not simple.

LEMma 4. Let $G$ be a finite non-solvable F-group. If G satisfies the oneprime power hypothesis, then $G / Z(G) \cong S L_{2}(4)$ or $S L_{2}(8)$.

Proof. Note that in Lemma 3 all groups satisfying (1)-(5) are solvable. So a non-solvable $F$-group satisfying the one-power prime hypothesis satisfies either (6) or (7). Suppose $G / Z(G) \cong P S L_{2}(q)$ and $q$ is odd. We want to obtain a contradiction. Recall that if $q \equiv 1[\bmod 4]$ we have $\operatorname{cs}^{*}(G / Z(G))=$ $\left\{q(q+1), q(q-1), \frac{1}{2} q(q+1), \frac{1}{2}(q-1)(q+1)\right\}$ and if $q \equiv 3[\bmod 4]$ we have $\operatorname{cs}^{*}(G / Z(G))=\left\{q(q+1), \frac{1}{2} q(q+1), \frac{1}{2}(q-1)(q+1), \frac{1}{2} q(q-1)\right\}$. Suppose that we have the first case.

By above discussion, for any $x \in G \backslash Z(G)$ we have $\left|x^{G}\right|=\left|(x Z(G))^{G / Z(G)}\right| a$, where $a \in\{1,2\}$. Note that, by Burnside's $p^{\alpha}$-Lemma, there is no non-trivial conjugacy class of $G / Z(G)$ of prime power size. If $b_{1}=c a_{1} \in \operatorname{cs}^{*}(G)$ and $b_{2}=c a_{2} \in \operatorname{cs}^{*}(G)$, where $c \in \operatorname{cs}^{*}(G / Z(G))$, then $c$ divides $\operatorname{gcd}\left(b_{1}, b_{2}\right)$ and so $\operatorname{gcd}\left(b_{1}, b_{2}\right)$ not a prime power. Thus $b_{1}=b_{2}$. Hence at most one conjugacy class size of $G$ is a multiple of $c$, for every $c \in \operatorname{cs}^{*}(G / Z(G))$. So $\operatorname{cs}^{*}(G)=\left\{q(q+1) a_{1}, q(q-1) a_{2}, \frac{1}{2} q(q+1) a_{3}, \frac{1}{2}(q-1)(q+1) a_{4}\right\}$, where $a_{1}$, $a_{2}, a_{3}, \quad a_{4} \in\{1,2\}$, and $\left|\operatorname{cs}^{*}(G)\right| \leq 4$. If $b_{1}=q(q+1) a_{1} \in \operatorname{cs}^{*}(G)$ and 
$b_{2}=q(q-1) a_{2} \in \operatorname{cs}^{*}(G)$, are distinct, then $2 q$ divides $\operatorname{ged}\left(b_{1}, b_{2}\right)$ and so $\left(b_{1}, b_{2}\right)$ not a prime power. So $b_{1}=b_{2}$. Thus there exist at most one conjugacy class size which is multiple of $q(q+1)$ and $q(q-1)$. Thus $\operatorname{cs}^{*}(G)=\left\{q(q+1) a_{1}, \frac{1}{2} q(q+1) a_{3}, \frac{1}{2}(q-1)(q+1) a_{4}\right\}$ and $\left|\operatorname{cs}^{*}(G)\right| \leq 3$. Now consider $b_{1}=q(q+1) a_{1} \in \operatorname{cs}^{*}(G)$ and $b_{4}=\frac{1}{2}(q-1)(q+1) a_{4} \in \operatorname{cs}^{*}(G)$, then $q+1$ divides $\operatorname{gcd}\left(b_{1}, b_{2}\right)$ and so $\left(b_{1}, b_{2}\right)$ not a prime power (if $q+1$ is a prime power, the since $q$ is odd we have $q+1=2^{k}$. Since by hypothesis $q=4 c+1$, we have $2^{k}-1=4 c+1$ and so $2^{k}=2(2 c+1)$, which is a contradiction). So $b_{1}=b_{4}$ and there exist at most one conjugacy class size which is multiple of $q(q+1)$ and $\frac{1}{2}(q-1)(q+1)$. Thus $\operatorname{cs}^{*}(G)=\left\{q(q+1) a_{1}\right.$, $\left.q(q-1) a_{2}\right\}$ and $\left|\operatorname{cs}^{*}(G)\right| \leq 2$ and $G / Z(G)$ is solvable, which is a contradiction. Similarly in the second case we can obtain a contradiction.

If $G / Z(G) \cong P G L_{2}(q)$, where $q$ is odd, then $\operatorname{cs}^{*}(G / Z(G))=\{q(q+1)$, $\left.q(q-1),(q-1)(q+1), \frac{1}{2} q(q+1), \frac{1}{2} q(q-1)\right\}$. As above, at most one conjugacy class size of $G$ is a multiple of $c$, for every $c \in \operatorname{cs}^{*}(G / Z(G))$, and $\operatorname{cs}^{*}(G)=\left\{q(q+1) a_{1}, q(q-1) a_{2},(q-1)(q+1) a_{3}, \frac{1}{2} q(q+1) a_{4}, \frac{1}{2} q(q-1) a_{5}\right\}$ and $\left|\operatorname{cs}^{*}(G)\right| \leq 5$. As above there exist at most one conjugacy class size which is multiple of $q(q+1)$ and $q(q-1)$. Thus $\operatorname{cs}^{*}(G)=\left\{q(q+1) a_{1}\right.$, $\left.(q-1)(q+1) a_{3}, \frac{1}{2} q(q+1) a_{4}, \frac{1}{2} q(q-1) a_{5}\right\}$ and $\left|\operatorname{cs}^{*}(G)\right| \leq 4$. Now either $q=4 k+1$ or $q=4 k+3$. Suppose $q=4 k+1$, then $q+1$ is not a prime power. Since $q+1$ divides $\operatorname{gcd}\left(b_{1}, b_{3}\right)$, where $b_{1}=q(q+1) a_{1}, b_{3}=$ $=(q-1)(q+1) a_{3}$ we have $b_{1}=b_{3}$ and $\operatorname{cs}^{*}(G)=\left\{q(q+1) a_{1}, \frac{1}{2} q(q+1) a_{4}\right.$, $\left.\frac{1}{2} q(q-1) a_{5}\right\}$. Hence by the main theorem of [13], $P G L_{2}(q) \cong G / Z(G) \cong$ $\cong P S L_{2}\left(2^{m}\right)$, a contradiction. Now suppose $q=4 k+3$. Then $2 q$ divides $\operatorname{gcd}\left(b_{4}, b_{5}\right)$, where $b_{4}=\frac{1}{2} q(q+1) a_{4}, b_{5}=\frac{1}{2} q(q-1) a_{3}$. Thus $b_{4}=b_{5}$ and $\operatorname{cs}^{*}(G)=\left\{q(q+1) a_{1},(q-1)(q+1) a_{3}, \frac{1}{2} q(q+1) a_{4}\right\}$. Hence by the main theorem of [13], $P G L_{2}(q) \cong G / Z(G) \cong P S L_{2}\left(2^{m}\right)$, a contradiction.

Finally suppose that $G / Z(G) \cong P S L_{2}\left(2^{n}\right)=P G L_{2}\left(2^{n}\right)$. Then $\operatorname{cs}^{*}(G / Z(G))=$ $=\left\{\left(2^{n}-1\right)\left(2^{n}+1\right), 2^{n}\left(2^{n}-1\right), 2^{n}\left(2^{n}+1\right)\right\}$. Therefore $\operatorname{cs}^{*}(G)=\left\{\left(2^{n}-1\right)\left(2^{n}+1\right) a_{1}\right.$, $\left.2^{n}\left(2^{n}-1\right) a_{2}, 2^{n}\left(2^{n}+1\right) a_{3}\right\}$. Since $2^{n}-1$ divides $\operatorname{gcd}\left(b_{1}, b_{2}\right)$, where $b_{1}=$ $=\left(2^{n}-1\right)\left(2^{n}+1\right) a_{1}, b_{2}=2^{n}\left(2^{n}-1\right) a_{2}$ and $2^{n}+1$ divides $\operatorname{gcd}\left(b_{1}, b_{3}\right)$, where $b_{3}=2^{n}\left(2^{n}+1\right) a_{3}$, we conclude that $2^{n}-1$ and $2^{n}+1$ are both prime power. Thus $n=2$ or $n=3$. Hence $G / Z(G) \cong S L_{2}(4)$ or $S L_{2}(8)$.

A generalization of Burnside $p^{\alpha}$-Lemma, which is due to Wielandt (see [1, Lemma 6]) states that if $x$ is a $p$-element, $p$ a prime, of $p$-power class size, then $x \in O_{p}(G)$, where $O_{p}(G)$ is the largest normal $p$-subgroup of $G$. Camina and Camina generalized this result and proved that all elements of prime power conjugacy class size are in the Fitting subgroup (see [5, Theorem 1]). We use this result in the following lemma. 
LEMMA 5. Let $G$ be a finite group satisfying the one-prime power hypothesis. If $G / Z(G)$ has no solvable normal subgroup, then $G$ is an F-group

Proof. Let $x, y$ be two non central elements of $G$ such that $C_{G}(x) \leq$ $\leq C_{G}(y)$. We have to prove $C_{G}(x)=C_{G}(y)$. If $C_{G}(x)<C_{G}(y)$, then $\left|x^{G}\right|$ and $\left|y^{G}\right|$ are distinct and $\left|y^{G}\right|$ divides $\left|x^{G}\right|$ and so $\operatorname{gcd}\left(\left|x^{G}\right|,\left|y^{G}\right|\right)=\left|y^{G}\right|$. Since $G$ satisfies he one-prime power hypothesis $\left|y^{G}\right|$ is a prime power. But since $\left|(y Z(G))^{G / Z(G)}\right|$ divides $\left|y^{G}\right|$ it follows that $\left|(y Z(G))^{G / Z(G)}\right|$ is also a prime power. Thus, by [5, Theorem 1], the Fitting subgroup of $G / Z(G)$ is non-trivial, which is a contradiction. Therefore $C_{G}(x)=C_{G}(y)$ and so $G$ is an $F$-group.

Corollary 1. Let $G$ be a finite simple group. Then $G$ satisfies the one-prime power hypothesis if and only if $G \cong S L_{2}(4)$ or $S L_{2}(8)$.

The converse of Lemma 4 is not true. For example if $G=S L_{2}(5)$, then $G / Z(G) \cong S L_{2}(4)\left(\cong A_{5}\right)$ and $\operatorname{cs}^{*}(G)=\{12,20,30\}$. In the following lemma we characterize a finite group whose factor group over its center is the alternating group on 5 letters, in terms of its conjugacy class sizes of the group. As a corollary we find sufficient conditions for a group to satisfy the one-prime power hypothesis. It is well known that in $A_{5}$ every element of order 3 or 5 , is self centralizing, that is $C_{A_{5}}(x)=\langle x\rangle$, for all $x \in A_{5}$ of order 3 or 5 . Also if $x$ is any element of order 2 in $A_{5}$, then $C_{A_{5}}(x)$ is a Sylow 2subgroup. Thus if $x \in P$ is any element of Sylow $p$-subgroup $P$ of $A_{5}$, $p \in\{2,3,5\}$, then $C_{G}(x)=P$.

We claim that if $x$ is a non-central element of a group $G$ such that $\left|\frac{C_{G}(x)}{Z(G)}\right|=p^{2}$, where $p$ is a prime, then $C_{G}(x)$ is abelian and thus there is no centralizer of any non-central element of $G$ strictly contained in $C_{G}(x)$. We use this fact in the proof of following lemma. To see the claim suppose, on the contrary that, $Z\left(C_{G}(x)\right)<C_{G}(x)$. Then $\frac{Z\left(C_{G}(x)\right)}{Z(G)}<\frac{C_{G}(x)}{Z(G)}$ and

$$
\left|C_{G}(x): Z\left(C_{G}(x)\right)\right|=\left|\frac{C_{G}(x)}{Z(G)}: \frac{Z\left(C_{G}(x)\right)}{Z(G)}\right|=p .
$$

Thus $C_{G}(x)$ is abelian, contradicting to $Z\left(C_{G}(x)\right)<C_{G}(x)$. Thus $Z\left(C_{G}(x)\right)=$ $=C_{G}(x)$ and so $C_{G}(x)$ is abelian. Now let $y$ be any non-central element of $G$ such that $C_{G}(y) \leq C_{G}(x)$. We want to show that $C_{G}(y)=C_{G}(x)$. Let $u \in C_{G}(x)$. Since $C_{G}(x)$ is abelian, $[u, v]=1$, for all $v \in C_{G}(x)$. In particular, since $y \in C_{G}(y) \leq C_{G}(x)$, we have $[u, y]=1$ and so $u \in C_{G}(y)$. Hence $C_{G}(y)=C_{G}(x)$, as required. This completes the proof of the claim. 
LEMMA 6. Let $G$ be a finite group such that $G / Z(G) \cong A_{5}$. Then $\operatorname{cs}^{*}(G)=\{12,15,20\}, \quad G^{\prime} \cong A_{5} \quad$ or $\quad \operatorname{cs}^{*}(G)=\{12,20,30\}, \quad G^{\prime} \cong S L_{2}(5)$. Therefore $G$ satisfies the one-prime power hypothesis if and only if $G^{\prime} \cong A_{5}$.

Proof. Let $x$ be a non central element of $G$. Then since $Z(G)<$ $<C_{G}(x)<G$, we have $|G: Z(G)|=\left|x^{G}\right|\left|C_{G}(x): Z(G)\right|$ and so $60=$ $=\left|x^{G}\right|\left|C_{G}(x): Z(G)\right|$. Since $\left|(x Z(G))^{G / Z(G)}\right|$ divides $\left|x^{G}\right|$, we have $\left|x^{G}\right| \in$ $\in\{12 a, 15 b, 20 c\}$, where $a, b, c$ are positive integers. If $\left|x^{G}\right|=12 a$, then $5=a\left|C_{G}(x): Z(G)\right|$ and so $a=1$, also $C_{G}(x)$ is abelian. If $\left|x^{G}\right|=20 c$, then $3=c\left|C_{G}(x): Z(G)\right|$ and so $c=1$, also $C_{G}(x)$ is abelian. If $\left|x^{G}\right|=15 b$, then $4=b\left|C_{G}(x): Z(G)\right|$ and so $b=1$ or 2 , also in the case $b=2, C_{G}(x)$ is abelian. Therefore $\left|x^{G}\right| \in\{12,15 b, 20\}$, where $b=1$ or 2 . We distinguish two cases.

CASE 1. Suppose that there exists $x \in G$ such that $\left|(x Z(G))^{G / Z(G)}\right|=15$ with $\frac{C_{G}(x)}{Z(G)}=C_{G / Z(G)}(x Z(G))$. Therefore

$$
\left|x^{G}\right|=\left|\frac{G}{Z(G)}: \frac{C_{G}(x)}{Z(G)}\right|=\left|\frac{G}{Z(G)}: C_{G / Z(G)}(x Z(G))\right|=15 .
$$

We want to prove for all $y \in G$ with $\left|(y Z(G))^{G / Z(G)}\right|=15$, we have $\frac{C_{G}(y)}{Z(G)}=C_{G / Z(G)}(y Z(G))$ and so $\left|y^{G}\right|=15$.

First of all, we know that $C_{G / Z(G)}(x Z(G))$ is a Sylow 2-subgroup of $G / Z(G)$ and, for every $y Z(G) \in C_{G / Z(G)}(x Z(G))$, we have $C_{G / Z(G)}(x Z(G))=$ $C_{G / Z(G)}(y Z(G))$. If $y Z(G) \in C_{G / Z(G)}(x Z(G))$, then $\frac{C_{G}(y)}{Z(G)} \leq C_{G / Z(G)}(y Z(G))=$ $C_{G / Z(G)}(x Z(G))=\frac{C_{G}(x)}{Z(G)}$. By the discussion in the paragraph preceding this Lemma, we have $C_{G}(x)=C_{G}(y)$. Therefore

$$
\frac{C_{G}(y)}{Z(G)}=\frac{C_{G}(x)}{Z(G)}=C_{G / Z(G)}(x Z(G))=C_{G / Z(G)}(y Z(G)) .
$$

Now suppose $y Z(G) \notin C_{G / Z(G)}(x Z(G))$. Then $C_{G / Z(G)}(y Z(G))$ is a Sylow 2subgroup of $G / Z(G)$ different from $C_{G / Z(G)}(x Z(G))$. Since $A_{5}$ acts transitively, by conjugation, on the set of its Sylow 2-subgroups, there exists $u \in G$ such that $u^{-1} x u Z(G) \in C_{G / Z(G)}(y Z(G))$. Hence $C_{G / Z(G)}(y Z(G))=$ $=C_{G / Z(G)}\left(u^{-1} x u Z(G)\right)$ and

$$
\frac{C_{G}(y)}{Z(G)} \leq C_{G / Z(G)}(y Z(G))=C_{G / Z(G)}(x Z(G))^{u}=\left(\frac{C_{G}(x)}{Z(G)}\right)^{u}=\frac{C_{G}\left(x^{u}\right)}{Z(G)} .
$$


Hence, by the discussion in the paragraph preceding this Lemma, $\frac{C_{G}(y)}{Z(G)}=\frac{C_{G}\left(x^{u}\right)}{Z(G)}$ and so $\frac{C_{G}(y)}{Z(G)}=C_{G / Z(G)}(y Z(G))$.

Thus we have proved that if $\left|(x Z(G))^{G / Z(G)}\right|=15$ and $\frac{C_{G}(x)}{Z(G)}=$ $=C_{G / Z(G)}(x Z(G))$, then for all $y \in G$ with $\left|(y Z(G))^{G / Z(G)}\right|=15$, we have $\frac{C_{G}(y)}{Z(G)}=C_{G / Z(G)}(y Z(G))$ and so $\left|y^{G}\right|=15$. Thus in this case $\operatorname{cs}^{*}(G)=$ $=\{12,15,20\}$.

Note that if $\left|x^{G}\right|=12$ or 20 , then $C_{G}(x)$ is abelian. Also if $\left|x^{G}\right|=15$ we saw that $C_{G}(x)$ is abelian. Hence there is no centralizer of any non-central element of $G$ strictly contained in $C_{G}(x)$. Thus $G$ is an $F$-group. Therefore by Lemma $3, G / Z(G) \cong P S L_{2}(4) \cong A_{5}$ and $G^{\prime} \cong S L_{2}(4) \cong A_{5}$. Note that in this case $G / Z(G) \cong P S L_{2}(5) \cong A_{5}$ but we can not have $G^{\prime} \cong S L_{2}(5)$, since otherwise 30 divides a conjugacy class size of $G$.

CASE 2. Now suppose that $\frac{C_{G}(x)}{Z(G)}<C_{G / Z(G)}(x Z(G))$, for all for $x \in G$ with $\left|(x Z(G))^{G / Z(G)}\right|=15$. Therefore

$$
\left|x^{G}\right|=\left|\frac{G}{Z(G)}: \frac{C_{G}(x)}{Z(G)}\right|=\left|(x Z(G))^{G / Z(G)}\right|\left|C_{G / Z(G)}(x Z(G)): \frac{C_{G}(x)}{Z(G)}\right|
$$

and so $\left|x^{G}\right|=30$ and also $\left|\frac{C_{G}(x)}{Z(G)}\right|=2$. Thus in this case $\operatorname{cs}^{*}(G)=$ $=\{12,20,30\}$.

Note that in the case $C_{G}(x)$ is abelian, for all $x \in G$. Thus $G$ is an $F$ group and by Lemma $3, G / Z(G) \cong P S L_{2}(5) \cong A_{5}$ and $G^{\prime} \cong S L_{2}(5)$.

Now we investigate the structure a finite group satisfying the oneprime power hypothesis whose factor group over its center is $S L_{2}(q)$.

LEMma 7. Let $G$ be a finite group such that $G / Z(G) \cong S$, where $S$ is a simple group with trivial Schur multiplier. Then $G \cong Z(G) \times S$. Therefore if $G / Z(G) \cong S L_{2}\left(2^{m}\right)$, where $m \geq 3$, then $G$ satisfies the one-prime power hypothesis if and only if $G=Z(G) \times S L_{2}(8)$.

Proof. By [18, Theorem 9.18 (1) and (6)], $\left|G^{\prime} \cap Z(G)\right|$ divides the order of the Schur multiplier of $S$. Therefore $G^{\prime} \cap Z(G)=1$. Now since $G^{\prime} Z(G) / Z(G)$ is a normal subgroup of a simple group $G / Z(G) \cong S$, it follows that $G=Z(G) \times G^{\prime} \cong Z(G) \times S$. Now since, by [10, Satz 25.7], the Schur multiplier of $S L_{2}\left(2^{m}\right), m \geq 3$, is trivial, and $S L_{2}\left(2^{m}\right), m \geq 3$, satisfies the 
one-prime power hypothesis if and only if $m=3$ (by the proof of Lemma 4) the other assertion follows.

Now we can prove Theorem 2.

Proof of Theorem 2. Suppose that $G$ satisfies the one-prime power hypothesis. Then, by Lemma $5, G$ is an $F$-groups and so, by Lemma 4 , $G / Z(G) \cong S L_{2}(q)$, where $q \in\{4,8\}$. Hence by Lemmas 6 and $7, G / Z(G) \cong$ $\cong S L_{2}(q) \cong G^{\prime}$, where $q \in\{4,8\}$. Let $H=Z(G) G^{\prime}$. Then $H=Z(G) \times G^{\prime}$ is a normal subgroup of $G$. Therefore $G^{\prime} \cong H / Z(G)$ is a normal subgroup of $G / Z(G)$ and so $H=G$. Hence $G=A \times G^{\prime}$, where $A$ is an abelian subgroup of $G$.

The converse is obvious.

We can find some information of the socle of finite groups satisfying the one-prime power hypothesis. Recall that the socle of a finite group $G$ is the subgroup generated by the minimal normal subgroups. It is well known that the socle of $G$ is a direct product of minimal normal subgroups.

Proposition 1. Let $G$ be a finite group satisfying the one-prime power hypothesis. Then the socle of $G$ is either abelian or is a direct product of a non abelian simple group and an abelian group.

Proof. We claim that if $N=S \times T$, where $S$ is a non abelian simple group, is a normal subgroup of $G$, then $Z(T) \neq 1$. The results follows from this claim. To prove the claim suppose $Z(T)=1$ and choose elements $x \in S$, $y \in T$ with coprime order. Then $C_{G}(x y)=C_{G}(x) \cap C_{G}(y) \leq C_{G}(x)$. If $C_{G}(x y)=C_{G}(x)$, then $T \leq C_{G}(x) \leq C_{G}(y)$ and so $y \in Z(T)=1$, a contradiction. Therefore $C_{G}(x y)<C_{G}(x)$ and thus $\left|x^{G}\right|$ is a proper divisor of $\left|(x y)^{G}\right|$. Since $G$ satisfies the one-prime power hypothesis, $\operatorname{gcd}\left(\left|x x^{G}\right|,\left|(x y)^{G}\right|\right)=\left|x^{G}\right|$ is a prime power. Since $\left|x^{S}\right|$ divides $\left|x^{N}\right|$ which is a divisor of $\left|x^{G}\right|$, it follows that $\left|x^{S}\right|$ is a prime power. Thus, by Burnside $p^{\alpha}$-Lemma, $S$ is not simple, a contradiction.

Now we consider finite groups satisfying the one-prime power hypothesis with simple socle, that is almost simple groups. First we need the following lemma. 
LEMMA 8. If $N$ be a normal subgroup of $G$ of index $p$, where $p$ is a prime, then for all $a \in \operatorname{cs}(N)$ either $a \in \operatorname{cs}(G)$ or $p a \in \operatorname{cs}(G)$. Thus if there exist $a, b \in \operatorname{cs}(N)$ such that $a<b,|\pi(\operatorname{gcd}(a, b))| \geq 2$, and $b \neq p a$, then $G$ does not satisfy the one-prime power hypothesis.

Proof. The first assertion is obvious. For the second assertion, suppose that $q r$ divide $\operatorname{gcd}(a, b)$, where $q$ and $r$ are primes. By above, $a, b \in \operatorname{cs}(G)$ or $a, p b \in \operatorname{cs}(G)$ or $p a, b \in \operatorname{cs}(G)$ or $p a, p b \in \operatorname{cs}(G)$. In any case $q r$ divides two distinct conjugacy class sizes of $G$. Thus $G$ does not satisfy the one-prime power hypothesis.

In the following lemma we prove that if $G$ is an almost simple group whose socle is $A_{n}$, then $G$ does not satisfy the one-prime power hypothesis.

LEMMA 9. The symmetric group on $n$ letters, $n \geq 5$, does not satisfy the one-prime power hypothesis. In particular if $A_{n} \leq G \leq \operatorname{Aut}\left(A_{n}\right)$, then $G$ does not satisfy the one-prime power hypothesis.

Proof. We have $\operatorname{cs}\left(S_{5}\right)=\{10,15,20,24,30\}$ and hence $S_{5}$, do not satisfy the one-prime power hypothesis. Suppose that $n \geq 6$. It is obvious that

$$
a:=\left|(12)(34)^{S_{n}}\right|=\frac{1}{2}\left[\frac{n(n-1)}{2} \frac{(n-2)(n-3)}{2}\right]
$$

and $b:=\left|(1234)^{S_{n}}\right|=\frac{n(n-1)(n-2)(n-3)}{24}$. Thus $a=3 b$ and so $\operatorname{gcd}(a, b)=b$. Since $b=\left|(1234)^{A_{n}}\right|$, we have that $b$ is not a prime power, by Burnside $p^{\alpha}$ Lemma. Hence $S_{n}$ does not satisfy the one-prime power hypothesis.

Now if $n \neq 6$, then $\operatorname{Aut}\left(A_{n}\right)=S_{n}$ and thus either $G=A_{n}$ or $G=S_{n}$. Hence, by above, $G$ does not satisfy the one-prime power hypothesis.

The outer automorphism group of $A_{6}$ is a Klein 4-group and if $A_{6} \leq G \leq \operatorname{Aut}\left(A_{6}\right)$, then $G$ is one of $A_{6}, S_{6}, P G L_{2}(9), M_{10}$, or $\operatorname{Aut}\left(A_{6}\right)$. In Atlas [8] notation, these are the groups $A_{6}, A_{6} .2_{1}, A_{6} .2_{2}, A_{6} .2_{3}$, and $S_{6} .2_{2}$, respectively.

We have $A_{6} \cong P S L_{2}(9)$ and $A=\operatorname{Aut}\left(A_{6}\right) \cong P \Gamma L_{2}(9)$, where $P \Gamma L_{2}(9)=$ $=\Gamma L_{2}(9) / Z\left(\Gamma L_{2}(9)\right)$ and $\Gamma L_{2}(9)$ is the group of all the semi-linear transformation of the vector space of dimension 2 over the Galois field with $q$ element. By [8], $A=P \Gamma L_{2}(9)$ has elements $x$ and $y$ of orders 2 and 5, respectively such that $\left|C_{A}(x)\right|=24$ and $\left|C_{A}(y)\right|=5$. Therefore $\left|x^{A}\right|=$ $=1440 / 24=60$ and $\left|y^{A}\right|=1440 / 5=288$ and $\left(\left|x^{A}\right|,\left|y^{A}\right|\right)=12$ is not a 
prime power. Thus Aut $\left(A_{6}\right)$ does not satisfies the one-prime power hypothesis. Now, by above, $S_{6}$ and, by Corollary $1, A_{6}, M_{10}$ do not satisfy the one-prime power hypothesis. Finally $\operatorname{cs}^{*}\left(P G L_{2}(9)\right)=\{36,45,72,80,90\}$ and so $P G L_{2}(9)$ does not satisfy the one-prime power hypothesis.

Now we consider sporadic simple groups.

LEMMA 10. If $S$ is a sporadic simple group and $S \leq G \leq \operatorname{Aut}(S)$, then $G$ does not satisfy the one-prime power hypothesis.

Proof. It is well known that the outer automorphism group of $S$ is either trivial or of order 2. If $S$ is one of the groups $M_{11}, M_{23}, M_{24}, C_{3}, C_{2}$, $\mathrm{Co}_{1}, \mathrm{Fi}_{23}, \mathrm{Th}, \mathrm{B}, \mathrm{M}, \mathrm{J}_{1}, L y, R u, J_{4}$, then the outer automorphism group of $S$ is trivial and so the result follows from Corollary 1. So suppose that $S$ is one of the groups $M_{12}, M_{22}, J_{2}, S u z, H S, M^{c} L, H e, F i_{22}, F i_{24}^{\prime}, H N, O^{\prime} N, J_{3}$. Using Atlas, in Table 1, the group $S$ and the order of centralizers of two elements $x, y \in S$, such that $a=\left|x^{S}\right|<b=\left|y^{S}\right|$ and $b \neq 2 a$, is given.

TABLE 1.

\begin{tabular}{|l|l|l|l|}
\hline \multicolumn{1}{|c|}{$S$} & \multicolumn{1}{|c|}{$|S|$} & $\left|C_{S}(x)\right|$ & $\left|C_{S}(y)\right|$ \\
\hline$M_{12}$ & $2^{6} \cdot 3^{3} \cdot 5 \cdot 11$ & 11 & 6 \\
\hline$M_{22}$ & $2^{7} \cdot 3^{2} \cdot 5 \cdot 7 \cdot 11$ & 11 & 7 \\
\hline$J_{12}$ & $2^{7} \cdot 3^{3} \cdot 5^{2} \cdot 7$ & 7 & 6 \\
\hline$S u z$ & $2^{13} \cdot 3^{7} \cdot 5^{2} \cdot 7 \cdot 11 \cdot 13$ & 13 & 11 \\
\hline$H S$ & $2^{9} \cdot 3^{2} \cdot 5^{3} \cdot 7 \cdot 11$ & 11 & 7 \\
\hline$M^{c} L$ & $2^{7} \cdot 3^{6} \cdot 5^{3} \cdot 7 \cdot 11$ & 11 & 5 \\
\hline$H e$ & $2^{10} \cdot 3^{3} \cdot 5^{2} \cdot 7^{3} \cdot 11$ & 17 & 14 \\
\hline$F i_{22}$ & $2^{17} \cdot 3^{9} \cdot 5^{2} \cdot 7 \cdot 11 \cdot 13$ & 14 & 13 \\
\hline$F i_{24}^{\prime}$ & $2^{21} \cdot 3^{16} \cdot 5^{2} \cdot 7^{3} \cdot 11 \cdot 13 \cdot 17 \cdot 23 \cdot 29$ & 29 & 23 \\
\hline$H N$ & $2^{14} \cdot 3^{6} \cdot 5^{6} \cdot 7 \cdot 11 \cdot 19$ & 22 & 19 \\
\hline$O^{\prime} N$ & $2^{9} \cdot 3^{4} \cdot 5 \cdot 7^{3} \cdot 11 \cdot 19 \cdot 31$ & 31 & 19 \\
\hline$J_{3}$ & $2^{7} \cdot 3^{5} \cdot 5 \cdot 17 \cdot 19$ & 19 & 17 \\
\hline
\end{tabular}

Hence, by Lemma 8, $G$ does not satisfy the one-prime power hypothesis. 
Investigating the structure of a group $G$ such that $B(G)$ has no cycle of length 4 seems a difficult task. For instance for a finite $p$-group $G, \Gamma(G)$ is a complete graph and $B(G)$ is a star graph, and there is no bound on the cardinality of the set of conjugacy class sizes of the group. In the case $\operatorname{diam}(\Gamma)=3$, the diameter of $\Gamma$, (recall that, by [7], $\operatorname{diam}(\Gamma(G)) \leq 3$ ) we are able to find such bound using a result of Kazarin [14]. For non-solvable groups we make the following conjecture.

Conjecture 1. If $G$ is a finite non-solvable group, then $B(G)$ has no cycle of length 4 if and only if $G=A \times S, S \cong S L_{2}(q)$, where $A$ is abelian, and $S \cong S L_{2}(q), q=4,8$.

By [7] we know that $\operatorname{diam}(\Gamma(G)) \leq 3$. Recall also that, by [2, Theorem 1], $n(\Gamma(G))$, the number of the connected components of $\Gamma(G)$ is not grater than 3 . In the following we find some information on a group satisfying the one-prime power hypothesis such that $\operatorname{diam}(\Gamma(G))=3$.

Proposition 2. Let $G$ satisfy the one-prime power hypothesis and assume $\Gamma(G)$ is connected with $\operatorname{diam}(\Gamma)=3$. Then $G$ is soluble and either $\pi(G)=3,|\operatorname{cs}(G)|=6$ or $\pi(G)=4,|\operatorname{cs}(G)|=9$.

Proof. By [14], $G=\left(A_{0} \times B_{0}\right) \times C_{0}$ where $A_{0}, B_{0}, C_{0}$ are groups with pairwise coprime orders, $A_{0}$ and $B_{0}$ are abelian and $A_{0} B_{0} / Z\left(A_{0} B_{0}\right)$ is a Frobenius group. Further, $\operatorname{cs}(G)=\left\{n, n a, n b \mid n \in \operatorname{cs}\left(C_{0}\right)\right\}$, where $a=$ $=\left|A_{0} /\left(A_{0} \cap Z\left(A_{0} B_{0}\right)\right)\right|$ and $b=\left|B_{0} /\left(B_{0} \cap Z\left(A_{0} B_{0}\right)\right)\right|$. For all $m \in \operatorname{cs}\left(C_{0}\right)$, with $m>1$, we have a path $a-m a-m-m b-b$. This implies that $a, b$ are distinct prime powers and $\operatorname{cs}\left(C_{0}\right)=\left\{1, u_{1}, \ldots, u_{r}\right\}$, where $u_{i}, i=1, \ldots, r$, are distinct prime powers other than $a, b$. Since $\Gamma$ is connected, $r \geq 1$. Since $C_{0}$ is a direct factor of $G$, it satisfies the one-prime power hypothesis. Also $n\left(\Gamma\left(C_{0}\right)\right) \leq 2$ and $\operatorname{diam}(\Gamma)=3$. So $r \leq 2$. If $r=1$, then $\operatorname{cs}\left(C_{0}\right)=\{1, u\}$ and so $\operatorname{cs}(G)=\{1, a, b, u, u a, u b\}$ and $|\operatorname{cs}(G)|=6$, see Figure 1 (I). If $r=2$, then $\operatorname{cs}\left(C_{0}\right)=\{1, u, v\}$ and $\operatorname{so} \operatorname{cs}(G)=\{1, a, b, u, v, u a, v a, u b, v b\}$ and $|\operatorname{cs}(G)|=9$, see Figure 1 (II).

Let $G=S_{3} \times E$, where $E$ is an extra special group of order $5^{3}$. Then $\operatorname{cs}(G)=\{1, a=2, b=3, u=5, u a=10, u b=15\}$. Let $H$ and $K$ be non abelian groups of order 10 and 21, respectively, and put $G=H \times K$. Then $\operatorname{cs}(H)=\{1,2,5\}, \operatorname{cs}(K)=\{1,3,7\}$ and $\operatorname{cs}(G)=\{1, a=3, b=7, u=2$, $v=5, u a=6, v a=15, u b=14, v b=35\}$. Therefore $\operatorname{diam}(\Gamma)=3$ and $G$ satisfies the one-prime power hypothesis. We note that there exists a group 
(I)

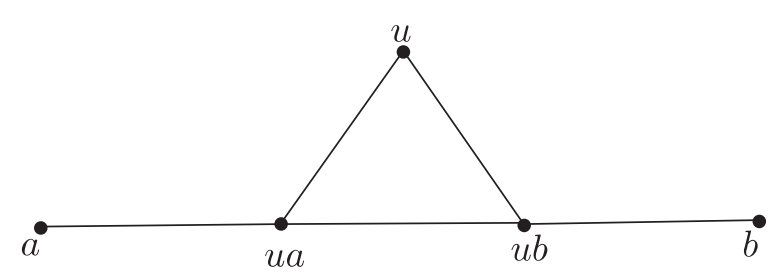

(II)

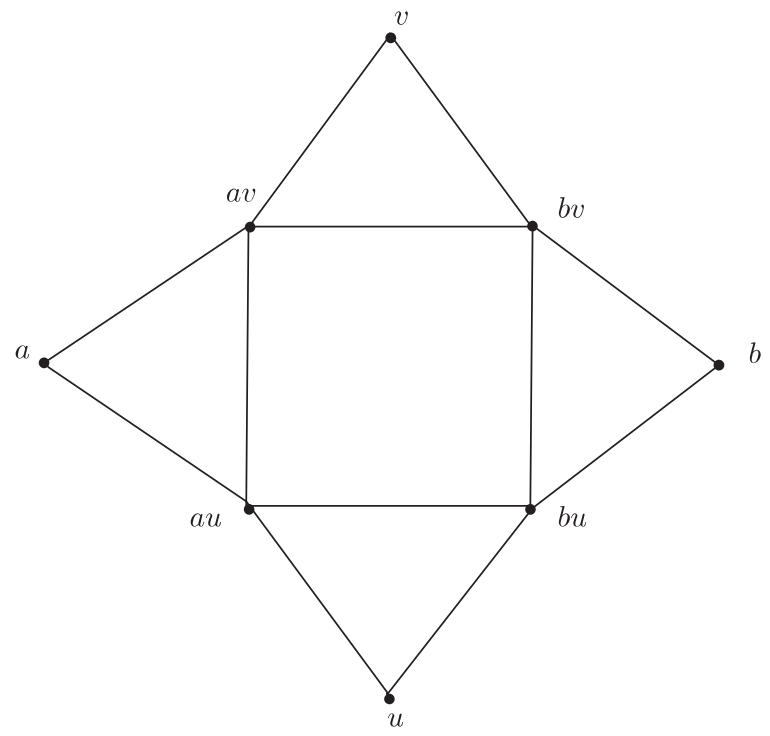

Fig. 1. - Graph $\Gamma(G)$ of a group $G$ satisfying the one-prime power hypothesis with diameter 3 .

whose bipartite graph have diameter 3 and does not satisfy the one-prime power hypothesis. For instance, let $G=H \times K$, where $H$ is a non abelian group of order 21 and $K$ is a dihedral group of order 30. Then $\operatorname{cs}(H)=\{1,3,7\}, \operatorname{cs}(K)=\{1,2,15\}$ and $\operatorname{socs}(G)=\{1,2,3,6,7,14,15,45,105\}$ and $\operatorname{diam}(\Gamma)=3$.

Acknowledgments. The author would like to express his gratitude to Silvio Dolfi for helpful comments. This work was done during the author's sabbatical leave. He is grateful to the School of Mathematics and Statistics, University of Western Australia for their warm hospitality and facilities provided, in particular Cheryl Praeger, for numerous, invaluable, conversations. The author thanks the Referee for valuable comments. 


\section{REFERENCES}

[1] R. BAER, Group elements of prime power index. Trans. Amer. Math. Soc., 75 (1953), pp. 20-47.

[2] E. A. Bertram - M. Herzog - A. Mann, On a graph related to conjugacy classes of groups, Bull. London Math. Soc., 22 (1990), pp. 569-575.

[3] D. Bubboloni - S. Dolfi - M. A. Iranmanesh - C. E. Praeger, On bipartite divisor graphs for group conjugacy class sizes, J. Pure Appl. Algebra, 213 (2009), pp. 1722-1734.

[4] C. CASOLO, Finite groups with small conjugacy classes. Manuscripta Math., 82 (1994), pp. 171-189.

[5] A. R. Camina - R. D. Camina, Implications of conjugacy class size, J. Group Theory, 1 (1998), pp. 257-269.

[6] A. R. CAmina - R. D. CAmina, Coprime conjugacy class sizes, Asian-European J. Math., 2 (2009), pp. 183-190.

[7] D. Chillag - M. Herzog - A. Mann, On the diameter of a graph related to conjugacy classes of groups, Bull. London Math. Soc., 113, No. 2 (1993), pp. 255-262.

[8] J. H. Conway - R. T. Curtis - S. P. Norton - R. A. Wilson, AtLAS of Finite Groups, Oxford Univ. Press (Oxford, 1985).

[9] S. DoLFI - E. JABARA, The structure of finite groups of conjugate rank 2, Bull. London Math. Soc. To appear.

[10] B. Huppert, Endliche Gruppen I, Springer-Verlag (Berlin, 1967).

[11] M. A. Iranmanesh - C. E. Praeger, Bipartite divisor graphs for integer subsets (submitted for publication).

[12] N. ITô, On finite groups with given conjugate types II, Osaka J. Math., 7 (1970), pp. 231-251.

[13] N. Itô, On Finite Groups with Given Conjugate Types. III, Math. Z., 117 (1970), pp. 267-271

[14] L. S. KAZARIN, On groups with isolated conjugacy classes, Izv. Vyssh. Uchebn Zaved. Mat., 25 (1981), pp. 40-45.

[15] M. L. LEWIS, Solvable groups having almost relatively prime distinct irreducible character degrees, J. Algebra, 174 (1995), pp. 197-216.

[16] M. FANG - P. ZHANG, Finite groups with graphs containing no triangles, J. Algebra, 264 (2003), pp. 613-619.

[17] J. Rebmann, F-Gruppen, Arch. Math., Vol. XXII (1971), pp. 225-230.

[18] M. SuzUki, Group Theory I, Springer-Verlag (New York, 1986).

Manoscritto pervenuto in redazione il 22 giugno 2009. 
